\title{
Chitosan/fucoidan multilayer nanocapsules as a vehicle for controlled release of bioactive compounds
}

\author{
Ana C. Pinheiro ${ }^{\mathrm{a}}$, Ana I. Bourbon ${ }^{\mathrm{a}}$, Miguel A. Cerqueira ${ }^{\mathrm{a}}$, Élia Maricato ${ }^{\mathrm{b}}$, Cláudia Nunes $^{\mathrm{b}}$, \\ Manuel A. Coimbra ${ }^{\mathrm{b}}$, António A. Vicente ${ }^{\mathrm{a}, *}$ \\ ${ }^{a}$ CEB-Centre of Biological Engineering, University of Minho, Campus de Gualtar, 4710-057 Braga, Portugal \\ b QOPNA, Department of Chemistry, University of Aveiro, 3810-193 Aveiro, Portugal
}

\section{A R T I C L E I N F O}

\section{Article history:}

Received 7 January 2014

Received in revised form 9 June 2014

Accepted 1 July 2014

Available online 16 July 2014

\section{Keywords:}

Nanocapsules

Layer-by-layer

Polyelectrolytes

Poly-L-lysine

Controlled release

\begin{abstract}
A B S T R A C T
Hollow multilayer nanocapsules were successfully prepared through layer-by-layer assembly of two bioactive polysaccharides, chitosan and fucoidan. The stepwise adsorption of 10 chitosan/fucoidan layers and the consequent formation of a multilayer film on polystyrene nanoparticles (used as templates) were followed through $\zeta$-potential measurement and the removal of the polystyrene core was confirmed by FTIR analysis. The chitosan/fucoidan nanocapsules morphology and size were evaluated by SEM and TEM, which showed that after the core removal, the nanocapsules maintained their spherical shape and a decrease of size occurred. A cationic bioactive compound, poly-L-lysine (PLL), was chosen to evaluate the loading and release behaviour of the nanocapsules. The chitosan/fucoidan nanocapsules showed a good capacity for the encapsulation and loading of PLL, which shows to be influenced by the initial PLL concentration and the method of encapsulation used. The results of fitting the linear superimposition model to the experimental data of PLL release suggest an anomalous behaviour, with one main polymer relaxation. The PLL release was found to be $\mathrm{pH}$-dependent: at $\mathrm{pH} 2$ relaxation is the governing phenomenon and at pH 7 Fick's diffusion is the main mechanism of PLL release.

Chitosan/fucoidan nanocapsules is a promising delivery system for water soluble bioactive compounds, such as PLL, showing a great potential of application in food and pharmaceutical industries
\end{abstract}

(c) 2014 Elsevier Ltd. All rights reserved.

\section{Introduction}

Nanoencapsulation systems exhibit high potential as carriers of bioactive substances. Their subcellular size allows relatively higher intracellular uptake and their permanence in circulation for longer, therefore extending their biological activity compared to micro-sized systems (Mora-Huertas, Fessi, \& Elaissari, 2010). Also, nanoencapsulation may be beneficial regarding improved stability and protection capability of labile substances against degradation factors (Preetz, Rübe, Reiche, Hause, \& Mäder, 2008). Layer-by-layer ( $\mathrm{LbL}$ ) deposition technique is one of the most powerful methods to create multilayer nanocapsules, which can be specially engineered with controlled sizes, composition, porosity, stability, surface functionality and colloidal stability and can be used as carriers for bioactive compounds. Also, the step-wise formation of multilayer nanocapsules allows introducing multiple

\footnotetext{
* Corresponding author. Tel.: +351253 604419, fax: +351253678986.

E-mail address: avicente@deb.uminho.pt (A.A. Vicente).
}

functionalities (Johnston, Cortez, Angelatos, \& Caruso, 2006). LbL assembly is based on the electrostatic interaction between oppositely charged polyelectrolytes alternatively adsorbed onto an appropriate template (Decher \& Schlenoff, 2003).

Core-shell nanoparticles can be produced through the deposition of polyelectrolyte layers onto colloidal nanoparticles; by subsequently removing the core (by dissolution or decomposition) from the core-shell structure, it is possible to obtain hollow nanocapsules with different properties, depending on the polyelectrolytes used (Cuomo, Lopez, Miguel, \& Lindman, 2010). Solid particles such as polystyrene, silica and $\mathrm{CaCO}_{3}$ are the most often used sacrificed templates for formation of capsules (Szczepanowicz et al., 2010). In addition, diameter, membrane thickness and permeability of hollow capsules prepared by LbL assembly can be easily controlled (Caruso, Caruso, \& Möhwald, 1998). Different bioactive compounds can be encapsulated in the hollow inner cavities of these nanocapsules and they can be released in a well-controlled manner (Li, Guo, Wen, \& Zhang, 2013).

Therefore, multilayer nanocapsules have promising applications in the release of bioactive compounds in the pharmaceutical and food industries; however for these applications to be possible, 
biofunctionality and the use of non-toxic materials are the most fundamental conditions to be met. Therefore, natural polyelectrolytes such as chitosan and fucoidan are competitive candidates as materials for the formation of multilayer nanocapsules. Chitosan is a cationic polysaccharide obtained by deacetylation of chitin which is the major constituent of exoskeleton of crustaceous animals. Chitosan is nontoxic, biodegradable, biocompatible and has intrinsic antimicrobial activity, inhibiting the growth of a wide variety of bacteria (Helander, Nurmiaho-Lassila, Ahvenainen, Rhoades, \& Roller, 2001; Shahidi, Arachchi, \& Jeon, 1999). Due to its promising properties, chitosan has been one of the most popular biopolymers for development of bioactive compounds delivery systems for a wide range of applications (Luo \& Wang, 2014). Fucoidan, an anionic sulphated polysaccharide found in brown algae display diverse biological activities, including antioxidant, anticoagulant, antibacterial, antiviral, anti-inflammatory and antitumor effects (Kusaykin et al., 2008).

Several techniques have been proposed to incorporate bioactive compounds within nanocapsules (Johnston et al., 2006). In general, the bioactive compound can be loaded during the preparation of capsules (adsorption of the bioactive compound to the surface of a particle that can be subsequently coated using the LbL technique) (Shu et al., 2010) or after the formation of the capsules (diffusion of bioactive compound from the surrounding medium in which the capsules are dispersed into the capsule) (Ye, Wang, Liu, \& Tong, 2005).

Poly-L-lysine (PLL) is a short cationic polypeptide composed of 27-33 identical L-lysine residues and is industrially produced by fermentation using Streptomyces albulus ssp. Lysinololymerus (Najjar, Kashtanov, \& Chikindas, 2007). This polypeptide exhibits a strong antimicrobial activity, is stable at high temperatures and under both acidic and alkaline conditions and is safe for human consumption (Najjar et al., 2007; Yoshida \& Nagasawa, 2003); therefore it is widely used as natural food preservative (Yamanaka \& Hamano, 2010). PLL inhibits the growth of a wide spectrum of microorganisms including Gram-positive and Gram-negative bacteria; it also has anti-phage activity (Hamano et al., 2007). Its mechanism of action against microbial growth is the electrostatic adsorption to the cell surface and subsequent interference with cell membranes (Najjar et al., 2007).

In this work, biodegradable hollow nanocapsules were developed through LbL assembly of chitosan and fucoidan. Nanocapsules were built through the alternate deposition of 10 chitosan/fucoidan layers on polystyrene (PS) nanoparticles (diameter $\approx 100 \mathrm{~nm}$ ), used as templates, followed by removal of the PS core. PLL was entrapped in the nanocapsules and its loading and release behaviour was evaluated.

\section{Materials and methods}

\subsection{Materials}

Dispersion of polystyrene nanoparticles with a diameter of $93.8 \pm 1.5 \mathrm{~nm}$ and a $\zeta$-potential of $-44.8 \pm 0.3 \mathrm{mV}$ was obtained from Polysciences, Inc. (Polybead ${ }^{\circledR}$ Polystyrene $0.10 \mu \mathrm{m}$ Microspheres, Warrington, PA, USA). Fucoidan with a $M_{W}=57,260$ was obtained from Sigma-Aldrich (Fucoidan from Fucus vesiculosus, St. Louis, MO, USA) and chitosan (deacetylation degree $\geq 95 \%$ ) was purchased from Golden-Shell Biochemical Co., Ltd. (Zhejiang, China). Lactic acid (90\%) was obtained from Acros Organics (Geel, Belgium) and ethanol, methanol and sulphuric acid were obtained from Panreac(Spain). Tetrahydrofuran(THF), 1,1-diphenyl-2-picrylhydrazyl (DPPH), hydrochloric acid and poly-L-lysine hydrobromide $\left(M_{W}\right.$ 1000-5000 Da) were purchased from Sigma-Aldrich (St. Louis, MO, USA).

\subsection{Determination of polysaccharides composition}

Fucoidan sample was hydrolyzed with $\mathrm{H}_{2} \mathrm{SO}_{4} 1 \mathrm{~mol} \mathrm{~L}^{-1}$ at $100^{\circ} \mathrm{C}$ during $2.5 \mathrm{~h}$ according to other authors (Selvendran, March, \& Ring, 1979). Neutral sugars were converted to alditol acetates, as previously described (Coimbra, Delgadillo, Waldron, \& Selvendran, 1996) and uronic acids were quantified through the 3-phenylphenol colorimetric method (Blumenkrantz \& AsboeHansen, 1973), modified by Coimbra et al. (1996).

Chitosan sample was hydrolyzed with $\mathrm{HCl} 6 \mathrm{~mol} \mathrm{~L}^{-1}$ at $100^{\circ} \mathrm{C}$ during $20 \mathrm{~h}$, followed by glucosamine conversion to alditol acetate by reduction with sodium borohydride and acetylation, as previously described (Coimbra et al., 1996). Alditol acetate derivatives were analysed by gas chromatography with a $30 \mathrm{~m}$ column DB225 (J\&W Scientific, Folsom, CA, USA) with i.d. and film thickness of $0.25 \mathrm{~mm}$ and $0.15 \mu \mathrm{m}$, respectively and using a flame ionization detector. The oven temperature program used was: initial temperature $200^{\circ} \mathrm{C}$, a rise in temperature at a rate of $40^{\circ} \mathrm{Cmin}^{-1}$ until $220^{\circ} \mathrm{C}$, standing for $7 \mathrm{~min}$, followed by a rate of $20^{\circ} \mathrm{C} \mathrm{min}-1$ until $230^{\circ} \mathrm{C}$ and maintained at this temperature for $1 \mathrm{~min}$. The injector and detector temperatures were, respectively, 220 and $230^{\circ} \mathrm{C}$. The flow rate of the carrier gas $\left(\mathrm{H}_{2}\right)$ was set at $1.7 \mathrm{~mL} \mathrm{~min}^{-1}$.

\subsection{Antioxidant activity of fucoidan}

The free-radical scavenging capacity of fucoidan was analysed using the 1,1-diphenyl-2-picrylhydrazyl (DPPH) test according to the method of Blois (1958), with some modifications. BHT and BHA were used as positive controls. Briefly, $0.2 \mathrm{~mL}$ of $\mathrm{MeOH}$ and $0.3 \mathrm{~mL}$ of the sample dissolved in $\mathrm{MeOH}$ (concentrations ranging from 0.05 to $\left.1.0 \mathrm{mg} \mathrm{mL}^{-1}\right)$ were mixed in a $10 \mathrm{~mL}$ test tube. DPPH $(2.5 \mathrm{~mL}$ of $75 \mu \mathrm{mol} \mathrm{L}^{-1}$ in $\mathrm{MeOH}$ ) were then added to achieve a final volume of $3.0 \mathrm{~mL}$. The solution was kept at room temperature for $30 \mathrm{~min}$ and the absorbance was measured at $517 \mathrm{~nm}$ (Blois, 1958).

The DPPH scavenging effect was calculated as follows:

scavevenging effect $(\%)=\frac{A_{0}-\left(A-A_{b}\right)}{A_{0}} \times 100$

where $A_{0}$ is the absorbance at $517 \mathrm{~nm}$ of DPPH without sample, $A$ is the absorbance at $517 \mathrm{~nm}$ of sample and DPPH and $A_{b}$ is the absorbance at $517 \mathrm{~nm}$ of sample without DPPH. The absorbance measurements were performed in Elisa Biotech Synergy HT(Biotek, USA).

\subsection{Antimicrobial activity of chitosan}

The antibacterial activity of chitosan was tested against two bacterial strains: Staphylococcus aureus (Gram-positive) and Escherichia coli (Gram-negative) by the disc agar diffusion test according to other authors (Bauer, Kirby, Sherris, \& Turck, 1966). Briefly, the chitosan solution $\left(1 \mathrm{mg} \mathrm{mL}^{-1}\right)$ was absorbed in sterilized filter paper discs $(0.6 \mathrm{~cm}$ in diameter) and placed on the lawn cultures of $S$. aureus and E. coli. The agar plates were incubated for $24 \mathrm{~h}$ at $37^{\circ} \mathrm{C}$ and diameters of the inhibitory zone of clearance $(\mathrm{cm})$ surrounding the discs were measured to estimate the antimicrobial activity. Sterile distilled water at the $\mathrm{pH}$ of the chitosan solution was used as control.

\subsection{Evaluation of the interactions between chitosan and fucoidan}

\subsubsection{Quartz crystal microbalance}

The adsorption behaviour of fucoidan and chitosan was evaluated using a quartz crystal microbalance (QCM 200, purchased from Stanford Research Systems, SRS, USA), equipped with AT-cut quartz crystals $(5 \mathrm{MHz})$ with optically flat polished chrome/gold electrodes on contact and liquid sides. 
Before carrying out the experiments, the crystal was cleaned by successive sonications $(40 \mathrm{KHz}, 30 \mathrm{~min})$ in ultra-pure water, ethanol and ultra-pure water, followed by drying with a gentle flow of nitrogen. Adsorption measurements were performed by alternate immersion of the crystal in chitosan $(\mathrm{pH} 3.0)$ and fucoidan ( $\mathrm{pH}$ 7.0) solutions, for $15 \mathrm{~min}$. The variations of the resonance frequency $(\Delta F)$ were simultaneously measured as a function of time and the analyses were performed at $25^{\circ} \mathrm{C}$, in triplicate.

\subsection{Preparation of nanocapsules}

The chitosan/fucoidan multilayers were assembled on the polystyrene nanoparticles by LbL deposition technique. The multilayer build-up was carried out according to other work (Ye et al., 2005) with some modifications. Briefly, $1 \mathrm{~mL}$ of chitosan solution $\left(1 \mathrm{mg} \mathrm{mL}^{-1}, \mathrm{pH} 3\right)$ in $1 \%$ of lactic acid was added dropwise to $1 \mathrm{~mL}$ of polystyrene particles' suspension $(0.5 \% \mathrm{v} / \mathrm{v})$ under gentle agitation and the mixture incubated for $15 \mathrm{~min}$ at room temperature. The excess of polysaccharide was removed by two repeated cycles of centrifugation $(12,000 \times \mathrm{g}, 20 \mathrm{~min}) /$ washing/redispersion in water/sonication $(40 \mathrm{KHz}, 60 \mathrm{~min})$. The washing steps after every deposition were performed to minimize occurrence of artefacts, i.e. polyelectrolyte aggregates formed in solution rather than on the core template, whereas the sonication step was implemented in order to disperse the nanocapsules in solution and minimize their aggregation. This procedure was repeated, this time using fucoidan solution ( $1 \mathrm{mg} \mathrm{mL}^{-1}, \mathrm{pH} 7$ ) as polyelectrolyte. The alternate deposition of chitosan and fucoidan was repeated until the deposition of 10 layers.

In order to remove the core of polystyrene particles, chitosan/fucoidan nanoparticles were treated with THF for $12 \mathrm{~h}$. The suspension was then centrifuged at $12,000 \times g$ for $15 \mathrm{~min}$ to remove THF. This procedure was repeated two times to assure complete removal of the polystyrene core and the resulting nanocapsules were washed three times with water, centrifuged and redispersed in distilled water.

\subsection{Characterization of nanoparticles and nanocapsules}

\subsection{1. $\zeta$-Potential measurements}

The $\zeta$-potential of coated polystyrene nanoparticles was determined by dynamic light scattering (DLS; Zetasizer Nano ZS, Malvern Instruments, UK). Each sample was analysed in a folded capillary cell. The $\zeta$-potential values are the average of nine successive measurements.

\subsubsection{Morphology}

The morphology of nanoparticles and nanocapsules was evaluated by scanning electron microscopy (SEM) (Nova NanoSEM 200, The Netherlands) with an accelerating voltage from 10 to $15 \mathrm{kV}$ and by transmission electron microscopy (TEM) (EM 902A, ZEISS, Germany).

SEM samples were prepared by depositing the suspension of nanoparticles or nanocapsules on Si slides. After drying the samples, they were coated with a thin gold layer (thickness of about $10 \mathrm{~nm}$ ). TEM samples were prepared by depositing the same suspensions on a carbon-coated copper grid. Before being analysed, samples were air-dried.

\subsubsection{Fourier transform infrared (FTIR) spectroscopy}

In order to confirm the removal of the polystyrene core, FTIR analyses were carried out with a Perkin Elmer 16 PC spectrometer (Perkin Elmer, Boston, MA, USA) in the wavenumber region of $600-4000 \mathrm{~cm}^{-1}$ using 16 scans for each sample. The nanocapsules were dried and then embedded in $\mathrm{KBr}$ pellets and pressed into $1 \mathrm{~mm}$ pellets for FTIR measurements.

\subsection{Poly-L-lysine encapsulation}

Two methods for PLL encapsulation were evaluated: adsorption of PLL in the polystyrene nanoparticles that were subsequently coated with 10 layers of chitosan/fucoidan and the core was removed (method 1) and diffusion of PLL into the capsules' interior after their formation (method 2). In method 1, a PLL solution was added to a suspension of polystyrene nanoparticles $(0.5 \% \mathrm{v} / \mathrm{v})$ and allowed to interact for $15 \mathrm{~min}$ (Shu et al., 2010). From preliminary results (by measuring the $\zeta$-potential of the nanoparticles coated with PLL at different incubation times), it was observed that 15 min of incubation was enough to guarantee the maximum PLL load capacity. For this method, different PLL concentrations, ranging from 0.25 to $2 \mathrm{mg} \mathrm{mL}^{-1}$, were tested in order to optimize the PLL loading.

In method 2, a PLL solution $\left(1 \mathrm{mg} \mathrm{mL}^{-1}\right)$ was added to the chitosan/fucoidan nanocapsules and allowed to diffuse into their interior during a $2 \mathrm{~h}$ incubation period (Ye et al., 2005).

The PLLencapsulation efficiency $(E E)$ and loading capacity $(L C)$ of the nanocapsules were determinate using the following equations (Shu et al., 2010):

$$
\begin{aligned}
& E E \%=\frac{\text { total protein }- \text { free protein }}{\text { total protein }} \times 100 \\
& L C \%=\frac{\text { total protein }- \text { free protein }}{\text { nanocapsules weight }} \times 100
\end{aligned}
$$

Free and bound PLL were separated by centrifugation $(14,000 \times g, 30 \mathrm{~min})$ using Amicon Ultra $(10 \mathrm{~K})$ (Millipore, Ireland) and the absorbance of the free PLL solution was measured at $215 \mathrm{~nm}$ (absorbance peak) (Elisa Biotech Synergy HT, Biotek, USA). The amount of free PLL in the supernatant was quantified from a previously prepared calibration curve of absorbance versus concentration of PLL.

\subsection{Determination of PLL release kinetics}

The in vitro release kinetics of PLL was performed by a dialysis method. The PLL-loaded nanocapsule suspension $(1.5 \mathrm{~mL})$ was added into a dialysis membrane (molecular weight cut-off $25 \mathrm{kDa}$; Cellu-Sep H1, Membrane filtration products, USA) that was subsequently placed into $40 \mathrm{~mL}$ of buffer solution (phosphate buffer for $\mathrm{pH} 7$ and $\mathrm{KCl}-\mathrm{HCl}$ buffer for $\mathrm{pH} 2$ ) under magnetic stirring.

At appropriate time intervals, $0.5 \mathrm{~mL}$ of supernatant were taken and $0.5 \mathrm{~mL}$ of fresh buffer were added to keep the volume of the release medium constant. The amount of PLL released from nanocapsules was evaluated by measuring the absorbance at $215 \mathrm{~nm}$ (absorbance peak) (Elisa Biotech Synergy HT, Biotek, USA). All release tests were run at least in triplicate.

In order to evaluate the release mechanism of PLL from chitosan/fucoidan nanocapsules a kinetic model that accounts for both Fickian and Case II transport effects in hydrophilic matrices, the linear superimposition model (LSM), was applied (Berens \& Hopfenberg, 1978):

$M_{t}=M_{t, F}+M_{t, R}$

where $M_{t, F}$ and $M_{t, R}$ are the contributions of the Fickian and relaxation processes, respectively, at time $t$. The purely Fickian process is described by:

$M_{t, F}=M_{\infty, F}\left[1-\frac{6}{\pi^{2}} \sum_{n=1}^{\infty} \frac{1}{n^{2}} \exp \left(-n^{2} k_{F} t\right)\right]$ 
where $M_{\infty, F}$ is the compound release at equilibrium and $k_{F}$ is the Fickian diffusion rate constant. Eq. (5) can be simplified using the first term of the Taylor series (Pinheiro, Bourbon, Quintas, Coimbra, \& Vicente, 2012b).

As for polymer relaxation, it is driven by the swelling ability of the polymer and is then related to the dissipation of stress induced by the entry of the penetrant and can be described as a distribution of relaxation times, each assuming a first order-type kinetic equation (Berens \& Hopfenberg, 1978).

$M_{t, R}=\sum_{i} M_{\infty, R_{i}}\left[1-\exp \left(-k_{R_{i}} t\right)\right]$

here, $M_{\infty, R_{i}}$ are the contributions of the relaxation processes for compound release and $k_{R_{i}}$ are the relaxation $i$ th rate constants. For most cases, there is only one main polymer relaxation that influences transport and thus the above equation can be simplified using $i=1$.

Therefore, the linear superimposition model for compound release from nanocapsules can be described by:

$\frac{M_{t}}{M_{\infty}}=X\left[1-\frac{6}{\pi^{2}} \exp \left(-k_{F} t\right)\right]+(1-X)\left[1-\exp \left(-k_{R} t\right)\right]$

where $X$ is the fraction of compound released by Fickian transport.

The experimental results were analysed by fitting Eqs. (5) (Fick's second law) and (7) (linear superimposition model) in order to assess the transport mechanism involved in the PLL release from nanocapsules at two different $\mathrm{pH}$ values.

\subsection{Statistical procedures}

\subsubsection{Non-linear regression analysis}

Statistical analyses were performed using the analysis of variance (ANOVA) procedure with SigmaPlot 11.0 software for Windows. Tukey's test was applied to detect differences of means, and $p<0.05$ was considered to be statistically significant.

The equations were fitted to data by non-linear regression, using a package of STATISTICA ${ }^{\mathrm{TM}}$ v7.0 (Statsoft. Inc, USA). The Levenberg-Marquardt algorithm for the least squares function minimization was used. The quality of the regressions was evaluated on the basis of the determination coefficient, $R^{2}$, the squared root mean square error, RMSE (i.e., the square root of the sum of the squared residues (SSE) divided by the regression degrees of freedom) and residuals visual inspection for randomness and normality. $R^{2}$ and SSE were obtained directly from the software. The precision of the estimated parameters was evaluated by the standardised halved width (SHW \%), which was defined as the ratio between the $95 \%$ standard error (obtained from the software) and the value of the estimate.

\section{Results and discussion}

\subsection{Polyelectrolytes characterization}

\subsubsection{Chemical composition}

The bioactive properties of polysaccharides usually depend on their chemical structure. Therefore, the chemical composition of fucoidan was performed and is listed in Table 1. Regarding the monosaccharide composition, the fucoidan sample is mainly composed of fucose (Fuc) and uronic acids. Minor amounts of other monosaccharide constituents such as galactose (Gal), xylose (Xyl), mannose (Man), glucose (Glc), and rhamnose (Rha) were also detected.

Other authors reported that fucoidan from $F$. vesiculosus is mainly composed by fucose, sulphate, and ash (Black, Dewar, \& Woodward, 1952), therefore the low percentage of sugars found in

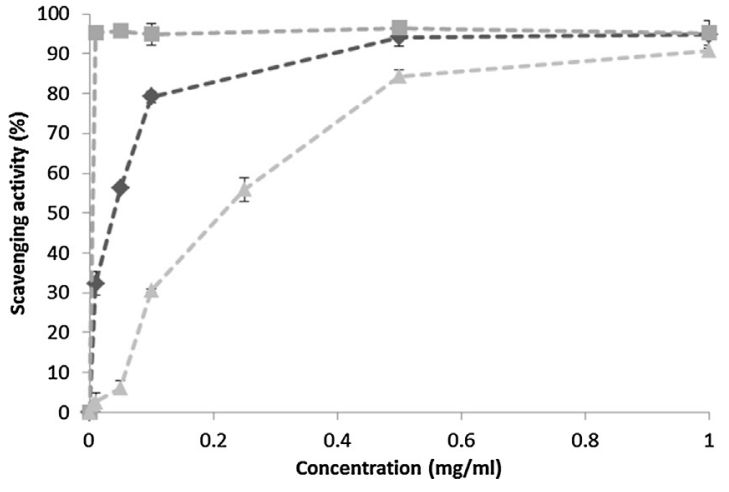

Fig. 1. Scavenging effects on DPPH radical of fucoidan $(\triangle)$ compared with the positive controls BHA $(\square)$ and BHT $(\diamond)$. Error bars represent the standard deviation obtained in the triplicate experiments.

this sample (about $58 \%$ ) is probably due to the presence of sulphate and ash.

The chitosan is mainly composed by D-glucosamine $\left(408.26 \pm 6.11 \mu \mathrm{g} \mathrm{mg}_{\text {sample }}{ }^{-1}\right)$, with only $7 \pm 2 \% \quad(\mathrm{w} / \mathrm{w})$ of $\mathrm{N}$-acetyl-Dglucosamine.

\subsubsection{Bioactive properties}

Chitosan and fucoidan are known for their bioactive properties (mainly antimicrobial and antioxidant activities, respectively) (Leceta, Guerrero, Ibarburu, Dueñas, \& de la Caba, 2013; Wang, Zhang, Zhang, Song, \& Li, 2010), being so, a preliminary study was conducted in order to verify the functional properties of these polysaccharides at concentrations as low as the ones typically used for the formation of nanocapsules through LbL methodology (Sun, Mutch, Lorenz, \& Chiu, 2005; Ye et al., 2005).

The antioxidant activity of fucoidan was investigated by the DPPH scavenging assay (Fig. 1). The reduction of DPPH (1,1diphenyl-2-picrylhydrazyl), a stable free radical, to DPPH-H after encountering a substance acting as a hydrogen atom donor can be used as an indicator of antioxidant activity, once when the stable non-radical form of DPPH is formed, a simultaneous change of solution's colour occurs from violet to pale yellow (Szabo, Idiţoiu, Chambre, \& Lupea, 2007). DPPH scavenging activity of fucoidan is concentration-dependent, and this polysaccharide exhibits a strong effect on inhibiting the formation of DPPH-H at concentrations between 0.1 and $1 \mathrm{mg} \mathrm{mL}^{-1}\left(\mathrm{IC}_{50}\right.$ of $\left.0.23 \mathrm{mg} \mathrm{mL}^{-1}\right)$. Moreover, at a concentration of $1 \mathrm{mg} \mathrm{mL}^{-1}$, fucoidan reaches a scavenging activity of $90.74 \%$. This value of scavenging activity is in agreement with the value obtained for crude fucoidan extracted from Undaria pinnatifida (86.80\% at $1 \mathrm{mg} \mathrm{mL}^{-1}$ ) (Mak, Hamid, Liu, Lu, \& White, 2013).

These results show that at a concentration of $1 \mathrm{mg} \mathrm{mL}^{-1}$ fucoidan presents a high antioxidant activity, therefore this was the concentration chosen for nanocapsules formation.

Although the antimicrobial activity of chitosan is well-know, the chitosan used is this work was tested in order to verify its antimicrobial activity at the concentration chosen for multilayer nanocapsules formation (the same concentration chosen for fucoidan: $1 \mathrm{mg} \mathrm{mL}^{-1}$ ). The same concentration of polysaccharides (in this case chitosan and fucoidan) was used based on other authors (Ye et al., 2005). Therefore, the antimicrobial activity of a chitosan solution against $S$. aureus and $E$. coli was evaluated using the disk diffusion technique (Fig. 2).

Chitosan exhibited antimicrobial activity against both $S$. aureus (Gram-positive) and E. coli (Gram-negative), although this activity was significantly $(p<0.05)$ higher against $S$. aureus. Chitosan has high antimicrobial activity against a wide range of microorganisms, including fungi, Gram-positive and Gram-negative bacteria, 
Table 1

Composition of fucoidan used to prepare the multilayer nanocapsules.

\begin{tabular}{|c|c|c|c|c|c|c|c|}
\hline \multicolumn{8}{|c|}{ Monosaccharide composition of fucoidan ( $\mu \mathrm{g}_{\text {monosaccharide }} \mathrm{mg}_{\text {sample }}{ }^{-1}$ ) } \\
\hline Rha & Fuc & Xyl & Man & Gal & Glc & Uronic acids & Total \\
\hline $1.1 \pm 0.0$ & $402.1 \pm 9.8$ & $29.8 \pm 2.3$ & $5.5 \pm 3.3$ & $36.0 \pm 4.1$ & $2.1 \pm 0.3$ & $91.7 \pm 4.1$ & 568.3 \\
\hline
\end{tabular}

however, several works showed that chitosan is more active against Gram-positive than against Gram-negative bacteria (Dutta, Tripathi, \& Dutta, 2012). The microbial target of protonated chitosan's actions would be the cytoplasmic membrane and cellular damage can lead to the disruption of the cellular integrity of the membrane. In the case of Gram-negative bacteria, the existence of an outer membrane protects the cells, once it acts as a barrier preventing chitosan from reaching the cytoplasmic membrane in the same extent as in the Gram-positive bacteria (Coma, Deschamps, \& Martial-Gros, 2003).

These results show that $1 \mathrm{mg} \mathrm{mL}^{-1}$ is also a suitable concentration for the chitosan solution, once this cationic polysaccharide exhibits a relevant antimicrobial activity against both Grampositive and Gram-negative bacteria.

\subsection{Interactions between chitosan and fucoidan}

Since LbL deposition is primarily governed by electrostatic interactions and in order to guarantee a correct deposition of chitosan and fucoidan layers, it is necessary to assure that these polyelectrolytes exhibit opposite charge. Once these polysaccharides are weak polyelectrolytes, their dissociation degree depends strongly on the solution's pH. The chitosan solution was found to have a maximum positive charge $(+62.5 \pm 4.9 \mathrm{mV})$ at $\mathrm{pH} 3$ and fucoidan solution exhibited a maximum negative charge $(-48.4 \pm 1.1 \mathrm{mV})$ at $\mathrm{pH} 7$.

The ability of these polyelectrolytes to form layers was evaluated through quartz crystal microbalance (QCM) analysis. Real-time build-up of chitosan and fucoidan nanolayered assemblies is reported in Fig. 3.

Fig. 3 shows the consecutive decrease of frequency after the alternate immersion of the crystal in chitosan and fucoidan solutions, which means that mass is being subsequently deposited and confirms the electrostatic interaction between these polyelectrolytes and the consequent formation of a stable multilayer structure. Also, from Fig. 3 it can be observed that the adsorption of fucoidan results in higher changes in frequency compared with the adsorption of chitosan, which is possibly due to the more pronounced hydrophilic character of fucoidan. Hydrophilic surfaces, such as fucoidan layer, are rough and can entrap solvents, which results in a more viscoelastic multilayered system and contributes

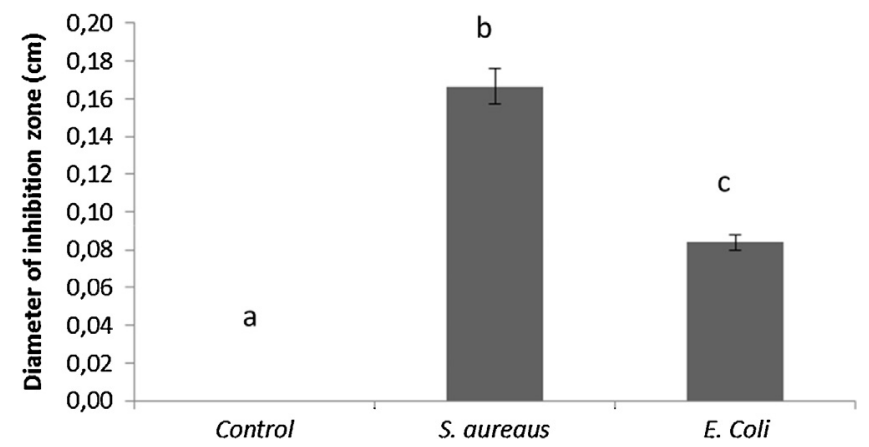

Fig. 2. Diameter of inhibition zones $(\mathrm{cm})$ of $S$. aureus and $E$. coli for a solution of $1 \mathrm{mg} \mathrm{mL} \mathrm{m}^{-1}$ of chitosan in the disk diffusion assay. Error bars represent the standard deviation obtained in the triplicate experiments. Different letters represent significantly different values according to the Tukey test $(p<0.05)$.

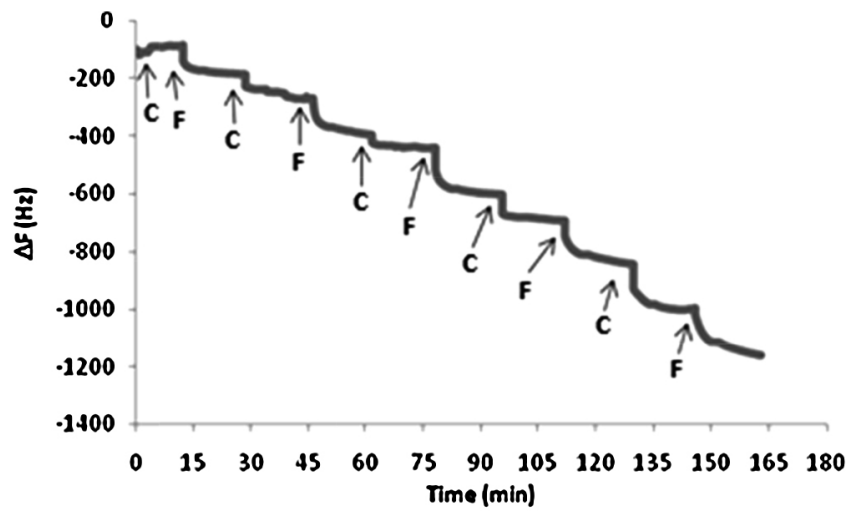

Fig. 3. Change in frequency as a function of time for chitosan (C, $1 \mathrm{mg} \mathrm{mL}^{-1}(\mathrm{w} / \mathrm{v})$, $\mathrm{pH} 3$ ) and fucoidan (F, $\left.1 \mathrm{mg} \mathrm{mL}^{-1}(\mathrm{w} / \mathrm{v}), \mathrm{pH} 7\right)$ adsorption.

to the mass increase sensed by the QCM and therefore to higher frequency changes. On the other hand, hydrophobic surfaces, such as chitosan layer, often do not wet and air can be entrapped, which results in smaller measured mass. A similar behaviour was observed for $\mathrm{\kappa}$-carrageenan/chitosan (Pinheiro et al., 2012a,b) and for alginate/chitosan multilayers (Martins, Mano, \& Alves, 2010).

The mass corresponding to the observed decrease of frequency also accounts for the water entrapped in the adsorbed layer (Indest et al., 2008), therefore a correct mass calculation can only be obtained for rigid thin films and was not performed in this work.

\subsection{Characterization of core-shell nanocapsules}

Once core-shell nanoparticles are the precursors of hollow nanocapsules, it is important to verify the successive deposition of polyelectrolytes layers on the polystyrene template and to follow shell formation.

\subsection{1. $\zeta$-Potential measurements}

The alternate deposition of chitosan and fucoidan on the polystyrene nanoparticles was monitored measuring $\zeta$-potential values at each assembly step (Fig. 4).

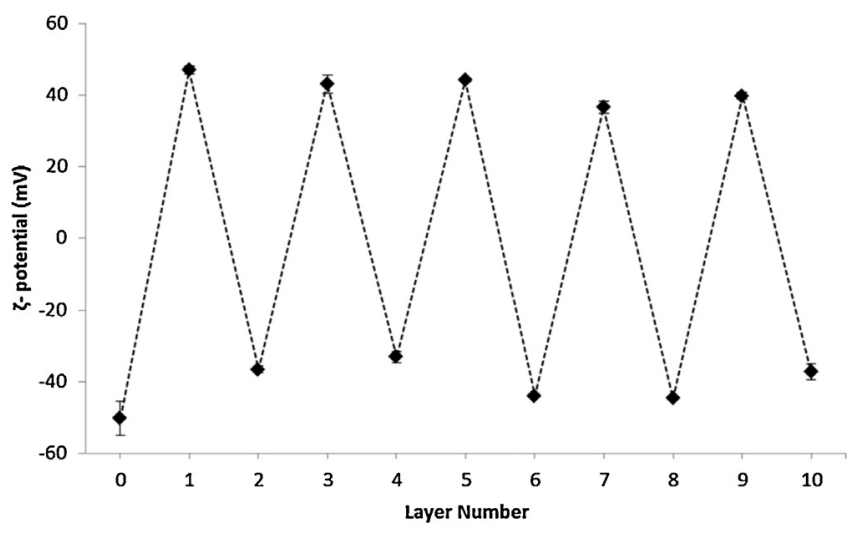

Fig. 4. $\zeta$-Potential change of polystyrene nanoparticles after each chitosan (even layers) or fucoidan (odd layers) deposition. The first point refers to the bare templates. Error bars represent the standard deviation obtained in the nine experiments. 


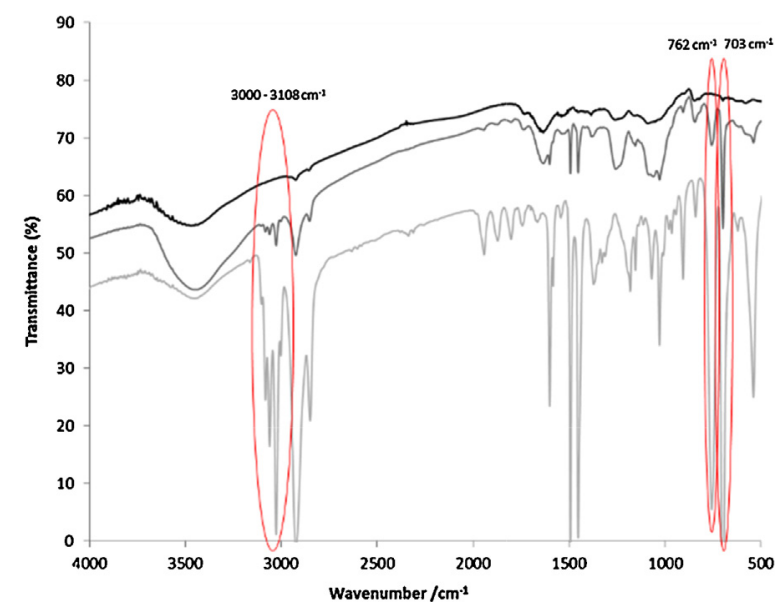

Fig. 5. FTIR spectra of polystyrene nanoparticles ( ${ }^{\prime}$ ), chitosan/fucoidan core-shell nanoparticles ( $)$ ) and hollow chitosan/fucoidan nanocapsules (-).

Polystyrene nanoparticles templates have a negative charge due to the presence of sulphate ester groups (Sun et al., 2005), so the initial step of polysaccharide multilayer construction consisted in the adsorption of the positively charged chitosan solution. $\zeta$ potential alternated between positive (more than $+30 \mathrm{mV}$ ) and negative (less than $-30 \mathrm{mV}$ ) values after deposition of chitosan and fucoidan, respectively, indicating the successfully deposition of these polysaccharides on the polystyrene nanoparticles up to 10 layers. Similar results were obtained by other authors for chitosan and alginate assembled on polystyrene (Ye et al., 2005) and $\mathrm{SiO}_{2}-\mathrm{NH}_{2}$ templates (Liu et al., 2012).
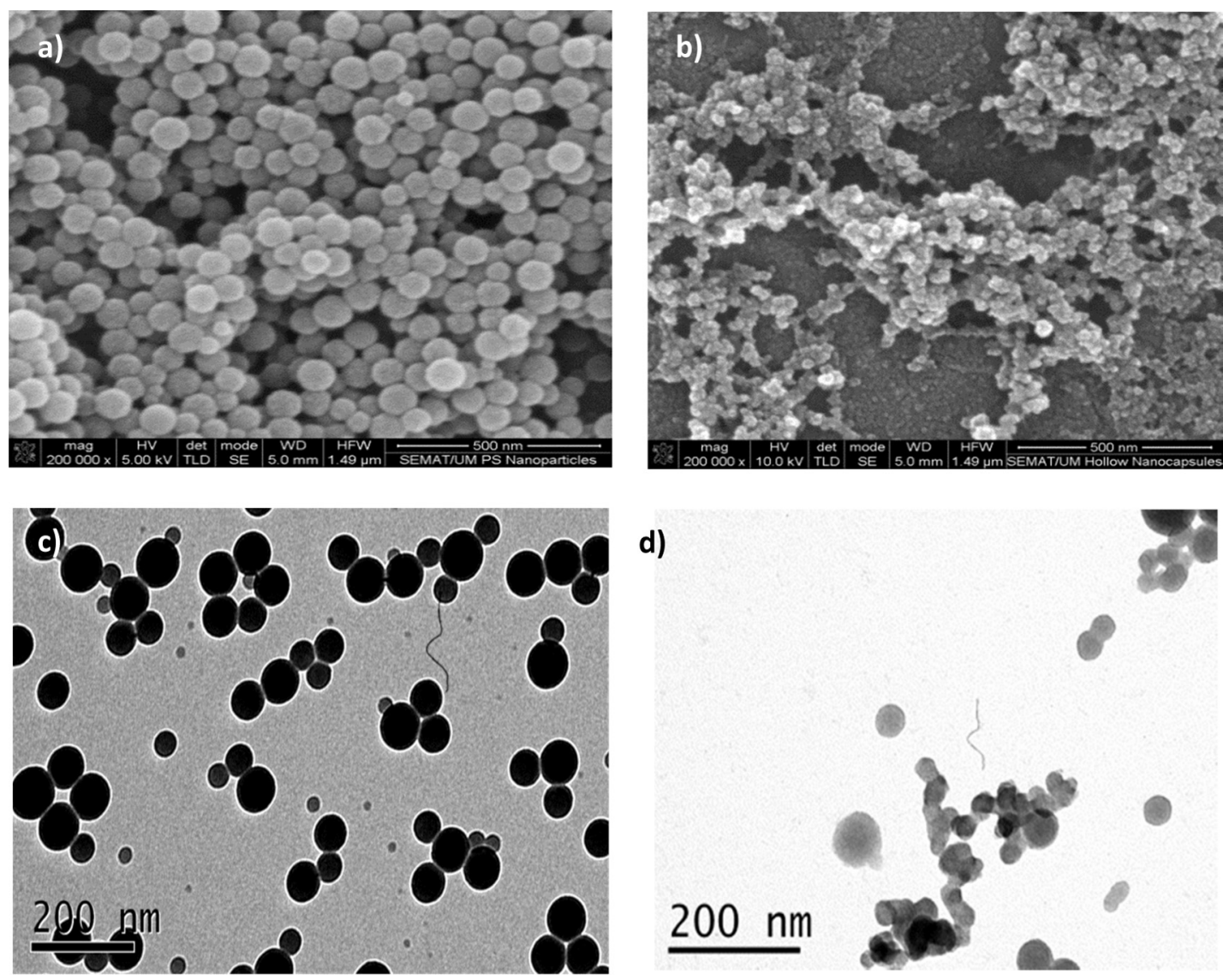

The $\zeta$-potential also gives considerable information on the stability of nanoparticles' suspensions, once particles with $\zeta$-potential values higher than $+30 \mathrm{mV}$ or lower than $-30 \mathrm{mV}$ are generally considered to be stable due to the strong repulsion forces that prevent the occurrence of aggregation phenomena among them (Liu et al., 2012). Therefore, the absolute values of $\zeta$-potential are large enough to indicate that nanoparticles of chitosan/fucoidan are stable, due to the strong electrostatic repulsion existing among them. Also, the magnitude of $\zeta$-potential values did not decrease with the subsequent layer deposition, suggesting that the mobility of nanoparticles did not decrease and therefore no significant aggregation occurred (Ye et al., 2005).

\subsection{Characterization of hollow nanocapsules}

After the removal of polystyrene core by repeated dipping in THF, hollow capsules can be obtained. The hollow capsules usually have the shape of the template and their stability and permeability features depend on the polyelectrolyte complex used (Cuomo et al., 2010).

\subsubsection{Fourier transform infrared (FTIR) spectroscopy}

In order to confirm the removal of the polystyrene core from chitosan/fucoidan nanoparticles, FTIR spectroscopy was carried out. IR spectra of the polystyrene nanoparticles, chitosan/fucoidan core-shell nanoparticles and hollow chitosan/fucoidan nanocapsules can be seen in Fig. 5 .

The characteristic bands of benzene residue in polystyrene appear between 3000 and $3108 \mathrm{~cm}^{-1}, 762$ and $703 \mathrm{~cm}^{-1}$ for both polystyrene nanoparticles and chitosan/fucoidan core-shell nanoparticles, indicating the presence of the polystyrene core.

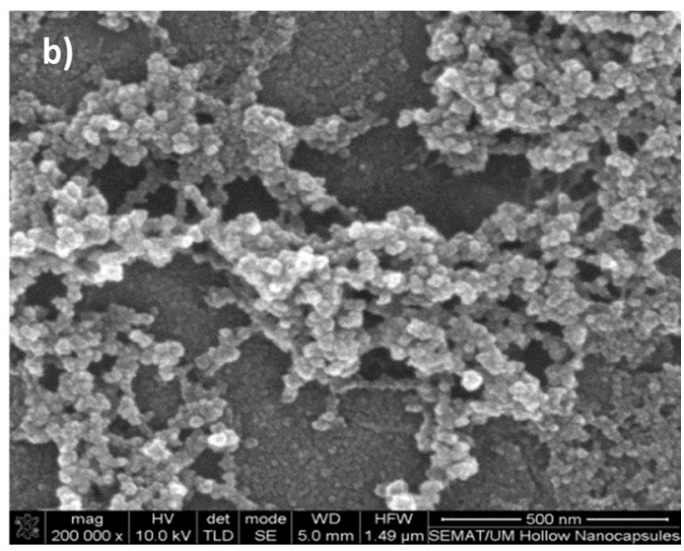

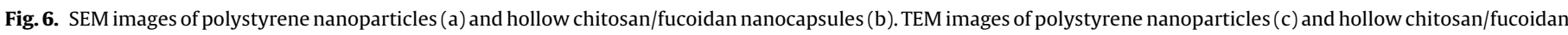
nanocapsules (d). 
However, after core removal by dissolution on THF, the characteristic polystyrene peaks disappear, confirming that the polystyrene core has been completely removed and that hollow nanocapsules have been formed.

\subsubsection{Morphology}

Morphological characteristics of polystyrene nanoparticles and hollow chitosan/fucoidan nanocapsules were examined using SEM and TEM and are presented in Fig. 6.

Microscopy images of the polystyrene template (Fig. 6a and c) show that particles are spherical and smooth and present an average diameter of approximately $90 \mathrm{~nm}$. After the deposition of chitosan and fucoidan layers, the dissolution of the supporting core by repeated dipping in THF resulted in the shrinking of the structure and on a consequent decrease of its size (to about $50 \mathrm{~nm}$ ), but they were found to maintain their spherical shape without rupturing of the wall as can be seen in Fig. 6b and d. Nanocapsules' diameter decreased due to the disorganization of the supporting core that led to a skink of the cyst wall (Liu et al., 2012). Contrary to the behaviour observed by other authors (Ye et al., 2005), the swelling of nanocapsules (i.e. increase of size) during the polystyrene core removal did not occur.

Fig. $6 \mathrm{c}$ and $\mathrm{d}$ shows that nanocapsules exhibit a reduced electron density compared with the core-shell nanoparticles, which is another indication of their hollow structure (Ye et al., 2005).

\subsubsection{Encapsulation and in vitro release of PLL}

Chitosan/fucoidan nanocapsules present per se bioactive potential due to the properties of their constituents; however, in order to evaluate the ability of these nanocapsules to encapsulate and release a bioactive compound, PLL was incorporated. The results obtained using two different encapsulation methods (method 1 -adsorption of PLL in the polystyrene nanoparticles and method 2-diffusion of PLL into the capsules' interior after their formation) showed that for the same PLL initial concentration $\left(1 \mathrm{mg} \mathrm{mL}^{-1}\right)$, the higher encapsulation efficiency $(E E)$ was obtained using method $1(E E=45.1 \pm 1.5 \%$ against $E E=26.01 \pm 5.5 \%$ for method 2$)$. The loadings achieved are generally higher when the bioactive compound is loaded during the preparation of capsules due to the high surface area of the capsule and due to the fact that, when the bioactive compound is loaded after capsules' formation, the maximum concentration of bioactive inside the capsules is often limited to the concentration in the solution (Johnston et al., 2006). Being so, method 1 was chosen as the method for PLL encapsulation. The mechanism of PLL encapsulation is based on the strong electrostatic interactions that are established between negatively charged polystyrene nanoparticles and positively charged PLL $(\zeta$ potential $=+26.0 \pm 1.5 \mathrm{mV}$ )

The encapsulation efficiency and loading capacity of nanocapsules as a function of PLL concentrations (from 0.25 to $2.0 \mathrm{mg} \mathrm{mL}^{-1}$ ) are represented in Fig. 7. This bioactive compound concentration range was also used by other authors for encapsulation of bovine serum albumin (BSA) in water soluble chitosan/dextran sulphate hollow nanocapsules (Shu et al., 2010).

The encapsulation efficiency $(E E)$ and the loading capacity $(L C)$ of the chitosan/fucoidan nanocapsules strongly depend on the initial PLL concentration used. The highest $E E$ and $L C$ values were observed at a PL concentration of $1 \mathrm{mg} \mathrm{mL}^{-1}$ and this was the concentration chosen for subsequent in vitro release experiments. The mechanisms of PLL release from chitosan/fucoidan nanocapsules were evaluated at $37^{\circ} \mathrm{C}$ (temperature within the human gastrointestinal system) and at two different $\mathrm{pH}$ values: 2 ( $\mathrm{pH}$ of the stomach) and 7 ( $\mathrm{pH}$ of the small intestine).

From Fig. 8a (profile release of PLL at $\mathrm{pH} 2$ ) and b (profile release of PLL at pH 7) it can be seen that the release of PLL occurs within the first two hours, at both $\mathrm{pH}$ values. This rapid release of the

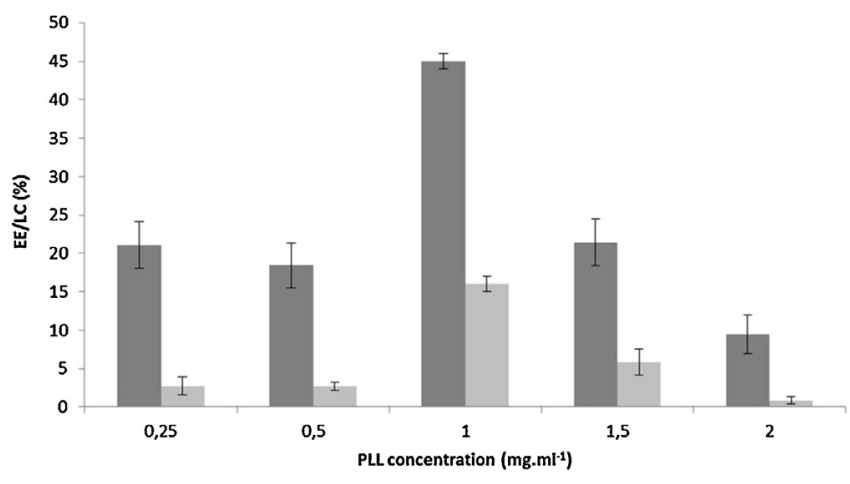

Fig. 7. Effect of PLL concentration on encapsulation efficiency ( $\square$ ) and loading capacity $(\square)$ of nanocapsules using method 1 .

bioactive compound can be found in other polymeric multilayer nanocapsules (Shu et al., 2010; Ye et al., 2005). Depending on the application, a slower release rate could be requided, however this can be achieved by addopting some strategies, such as the addition of a crosslinking agent (Ye et al., 2005). Even though, the release test performed just with free PLL (results not shown) showed a burst PLL release, suggesting that the nanocapsules can slow the release of PLL.

Also, Fig. 8 shows that nanocapsules present a pH-dependent release pattern. In order to evaluate the mechanism of PLL release from nanocapsules at $\mathrm{pH} 2$ and $\mathrm{pH} 7$, the exprimental results were analysed by fitting Fick's second law (Eq. (5)) and LSM (Eq. (7)). For both pH conditions, LSM fitting curves showed a better description of the experimental data (Fig. 8), indicating that this transport mechanism cannot only be described by Brownian motion of PLL in the nanoparticles, i.e. it does not strictly follow Fick's behaviour, but is governed by both Fickian and Case II transport, with only one main relaxation of the nanocapsules.

Table 2 presents the regression analysis results of the LSM fitting, showing that this model adequately describes the experimental data with relatively good regression quality $\left(R^{2}>0.90\right)$ and that almost all parameters were estimated with good precision.

The parameter $X$ is defined as the Fick's diffusion contribution to the total release from the nanocapsules $\left(M_{\infty, F} / M_{t}\right)$ and it can be observed from Table 2 that for $\mathrm{pH} 2 X<0.5$ and for $\mathrm{pH} 7$ $X>0.5$, which indicates that at $\mathrm{pH} 2$ relaxation is the governing phenomenon and at $\mathrm{pH} 7$ Fick's diffusion is the main release mechanism. These results may be related to the magnitude of the electrostatic interactions between the polyelectrolytes at different $\mathrm{pH}$ values. The pKa value of the anionic sulphate groups of fucoidan is around $\mathrm{pH} \mathrm{2,} \mathrm{therefore,} \mathrm{at} \mathrm{this} \mathrm{pH}$ these groups are half protonated and consequently partially uncharged, which means that electrostatic interactions between fucoidan and PLL or chitosan are weaker. This leads to the loosening of nanocapsules' structure and promotes the release of PLL due to polymer relaxation. Also the relaxation rate constant $\left(k_{R}\right)$ decreased with the increase of $\mathrm{pH}$, supporting this hypothesis. On the contrary, at $\mathrm{pH} 7$, most of the amino groups of chitosan are in the $-\mathrm{NH}_{2}$ form (once the pKa of chitosan is around 6.5) and the sulphate groups are in the $-\mathrm{SO}^{3-}$ form, therefore also at this $\mathrm{pH}$, weak electrostatic interactions between the polyelectrolytes and relaxation as the dominant mechanism would be expected. However, comparing with $\mathrm{pH} 2$, this behaviour is not so accentuated, and the main release mechanism is Fick's diffusion, which suggests that other phenomena, besides the electrostatic interactions, may be involved. As for the Fickian rate constant $\left(k_{F}\right)$, this parameter increased with $\mathrm{pH}$, reflecting the predominance of Fick's behaviour at $\mathrm{pH}$ 7. A similar behaviour was observed for the release of methylene blue from a 

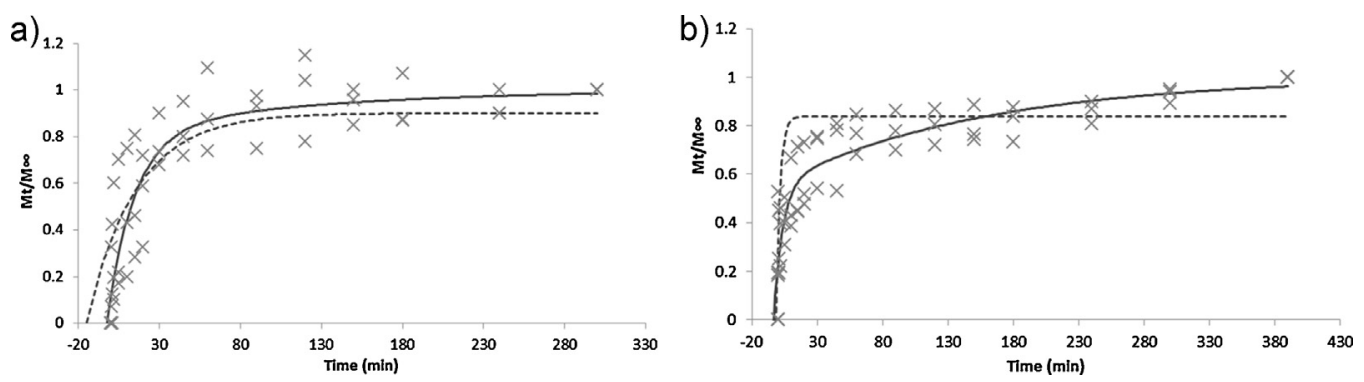

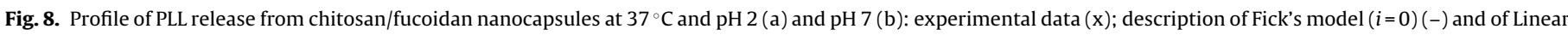
Superimposition Model ( $i=1)(--)$.

Table 2

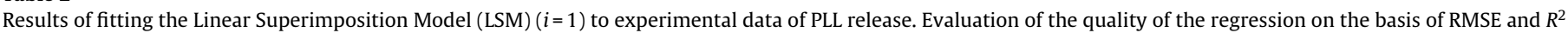
Estimates' precision is evaluated using the SHW\% (in parenthesis).

\begin{tabular}{|c|c|c|c|c|c|}
\hline $\mathrm{pH}$ & RMSE & $R^{2}$ & $X$ & $k_{F}\left(\min ^{-1}\right)$ & $k_{R}\left(\min ^{-1}\right)$ \\
\hline 2 & 0.160 & 0.903 & $0.299(78.40 \%)$ & 0.008 (148.01\%) & $0.059(38.40 \%)$ \\
\hline 7 & 0.113 & 0.915 & $0.565(21.42 \%)$ & 0.145 (106.97\%) & $0.033(46.30 \%)$ \\
\hline
\end{tabular}

nanolayered coating composed of $\kappa$-carrageenan (also a sulphated anionic polysaccharide) and chitosan (Pinheiro et al., 2012b).

\section{Conclusions}

This work demonstrated that layer-by-layer deposition of chitosan/fucoidan layers on a sacrificial template allows the production of biodegradable hollow nanocapsules with potential antioxidant and antimicrobial activity and with great potential to act as a controlled delivery system for bioactive compounds. Chitosan/fucoidan nanoparticles showed to be stable and they were found to maintain their spherical shape without rupturing of the wall after the core removal. Poly-L-lysine, a cationic bioactive compound, was encapsulated in the nanocapsules and its loading and release behaviour was evaluated. The nanocapsules showed a good capacity for the encapsulation and loading of PLL. The results of fitting the linear superimposition model to the experimental data of PLL release suggested an anomalous behaviour, with one main polymer relaxation, and PLL release was found to be $\mathrm{pH}$-dependent. This work allows clarifying release mechanisms involved at two different $\mathrm{pH}$ values found within a human gastrointestinal tract, which is of utmost importance to control the rate of release of bioactive compounds under those circumstances.

Due to the bioactive and non-toxic nature of chitosan/fucoidan nanocapsules and due to their responsiveness at different $\mathrm{pHs}$, these capsules are envisaged as a nanocarrier system to protect and release bioactive compounds for food and pharmaceutical applications.

\section{Acknowledgments}

The authors Ana I. Bourbon, Miguel A. Cerqueira, Élia Maricato, and Cláudia Nunes are recipient of fellowships from the Fundação para a Ciência e Tecnologia, POPH-QREN and FSE (FCT, Portugal) through grants, SFRH/BD/73178/2010, SFRH/BPD/72753/2010, SFRH/BD/87245/2012 and SFRH/BPD/46584/2008, respectively. The authors thank the FCT Strategic Project PEst-OE/EQB/LA0023/2013 and the project "BioInd-Biotechnology and Bioengineering for improved Industrial and Agro-Food processes; REF. NORTE-07-0124-FEDER-000028 Co-funded by the Programa Operacional Regional do Norte (ON.2-O Novo Norte), QREN, FEDER. Also, thanks are due to Fundação para a Ciência e a Tecnologia (FCT, Portugal), European Union, QREN, FEDER and COMPETE for funding the QOPNA research unit (project
PEst-C/QUI/UI0062/2013; FCOMP-01-0124-FEDER-037296). The authors would like to acknowledge to Rui Fernandes from IBMC, University of Porto for assistance in TEM analysis and to Edith Ariza from SEMAT/UM by the support in SEM analysis.

\section{References}

Bauer, A., Kirby, W., Sherris, J., \& Turck, M. (1966). Antibiotic susceptibility testing by a standardized single disk method. American Journal of Clinical Pathology, 45 493-496.

Berens, A. R., \& Hopfenberg, H. B. (1978). Diffusion and relaxation in glassy polymer powders. 2. Separation of diffusion and relaxation parameters. Polymer, 19(5) 489-496.

Black, W. A. P., Dewar, E. T., \& Woodward, F. N. (1952). Manufacture of alga chemicals. IV.-Laboratory-scale isolation of fucoidan from brown marine algae. Journal of the Science of Food and Agriculture, 3, 122-129.

Blois, M. S. (1958). Antioxidant determinations by the use of a stable free radical. Nature, 181(4617), 1199-1200.

Blumenkrantz, N., \& Asboe-Hansen, G. (1973). New method for quantitative determination of uronic acids. Analytical Biochemistry, 54(2), 484-489

Caruso, F., Caruso, R. A., \& Möhwald, H. (1998). Nanoengineering of inorganic and hybrid hollow spheres by colloidal templating. Science, 282(5391), 1111-1114.

Coimbra, M. A., Delgadillo, I., Waldron, K. W., \& Selvendran, R. R. (1996). Isolation and analysis of cell wall polymers from olive pulp. In H. Linskens, \& J. Jackson (Eds.), Plant cell wall analysis (Vol. 17) (pp. 19-44). Berlin/Heidelberg: Springer

Coma, V., Deschamps, A., \& Martial-Gros, A. (2003). Bioactive packaging materials from edible chitosan polymer-antimicrobial activity assessment on dairyrelated contaminants. Journal of Food Science, 68(9), 2788-2792.

Cuomo, F., Lopez, F., Miguel, M. G., \& Lindman, B. r. (2010). Vesicle-templated layer-by-layer assembly for the production of nanocapsules. Langmuir, 26(13) 10555-10561.

Decher, G., \& Schlenoff, J. B. (2003). Polyelectrolyte multilayers, an overview. Weinheim: Wiley-VCH Verlag GmbH \& Co. KGaA.

Dutta, J., Tripathi, S., \& Dutta, P. K. (2012). Progress in antimicrobial activities of chitin, chitosan and its oligosaccharides: A systematic study needs for food applications. Food Science and Technology International, 18(1), 3-34.

Hamano, Y., Nicchu, I., Shimizu, T., Onji, Y., Hiraki, J., \& Takagi, H. (2007). $\varepsilon$ Poly-L-lysine producer, streptomyces albulus, has feedback-inhibition resistant aspartokinase. Applied Microbiology and Biotechnology, 76(4), 873-882.

Helander, I. M., Nurmiaho-Lassila, E. L., Ahvenainen, R., Rhoades, J., \& Roller, S. (2001). Chitosan disrupts the barrier properties of the outer membrane of Gramnegative bacteria. International Journal of Food Microbiology, 71(2-3), 235-244.

Indest, T., Laine, J., Ribitsch, V., Johansson, L.-S., Stana-Kleinschek, K., \& Strnad, S. (2008). Adsorption of chitosan on pet films monitored by quartz crystal microbalance. Biomacromolecules, 9(8), 2207-2214.

Johnston, A. P. R., Cortez, C., Angelatos, A. S., \& Caruso, F. (2006). Layer-by-layer engineered capsules and their applications. Current Opinion in Colloid E Interface Science, 11(4), 203-209.

Kusaykin, M., Bakunina, I., Sova, V., Ermakova, S., Kuznetsova, T., Besednova, N., Zaporozhets, T., \& Zvyagintseva, T. (2008). Structure, biological activity, and enzymatic transformation of fucoidans from the brown seaweeds. Biotechnology Journal, 3(7), 904-915.

Leceta, I., Guerrero, P., Ibarburu, I., Dueñas, M. T., \& de la Caba, K. (2013). Characterization and antimicrobial analysis of chitosan-based films. Journal of Food Engineering, 116(4), 889-899. 
Li, G. Guo, L., Wen, O, \& Zhang, T. (2013). Thermo- and pH-sensitive ionic-crosslinked hollow spheres from chitosan-based graft copolymer for 5 fluorouracil release. International Journal of Biological Macromolecules, 55(0), 69-74.

Liu, Y., Yang, J., Zhao, Z., Li, J., Zhang, R., \& Yao, F. (2012). Formation and characterization of natural polysaccharide hollow nanocapsules via template layer-by-layer self-assembly. Journal of Colloid and Interface Science, 379(1), 130-140.

Luo, Y., \& Wang, Q. (2014). Recent development of chitosan-based polyelectrolyte complexes with natural polysaccharides for drug delivery. International Journal of Biological Macromolecules, 64(0), 353-367.

Mak, W., Hamid, N., Liu, T., Lu, J., \& White, W. L. (2013). Fucoidan from New Zealand Undaria pinnatifida: Monthly variations and determination of antioxidant activities. Carbohydrate Polymers, 95(1), 606-614.

Martins, G. V., Mano, J. F., \& Alves, N. M. (2010). Nanostructured self-assembled films containing chitosan fabricated at neutral $\mathrm{pH}$. Carbohydrate Polymers, $80(2)$ 570-573.

Mora-Huertas, C. E., Fessi, H., \& Elaissari, A. (2010). Polymer-based nanocapsules for drug delivery. International Journal of Pharmaceutics, 385(1-2), 113-142.

Najjar, M. B., Kashtanov, D., \& Chikindas, M. L. (2007). $\varepsilon$-Poly-L-lysine and nisin A act synergistically against Gram-positive food-borne pathogens Bacillus cereus and Listeria monocytogenes. Letters in Applied Microbiology, 45(1), 13-18.

Pinheiro, A. C., Bourbon, A. I., Medeiros, B. G. d. S., da Silva, L. H. M., da Silva, M. C. H. Carneiro-da-Cunha, M. G., Coimbra, M. A., \& Vicente, A. A. (2012). Interactions between $\kappa$-carrageenan and chitosan in nanolayered coatings-Structural and transport properties. Carbohydrate Polymers, 87(2), 1081-1090.

Pinheiro, A. C., Bourbon, A. I., Quintas, M. A. C., Coimbra, M. A., \& Vicente, A. A. (2012). K-carrageenan/chitosan nanolayered coating for controlled release of a model bioactive compound. Innovative Food Science \& Emerging Technologies, 16(0), 227-232.

Preetz, C., Rübe, A., Reiche, I., Hause, G., \& Mäder, K. (2008). Preparation and characterization of biocompatible oil-loaded polyelectrolyte nanocapsules. Nanomedicine: Nanotechnology, Biology, and Medicine, 4(2), 106-114.
Selvendran, R. R., March, J. F., \& Ring, S. G. (1979). Determination of aldoses and uronic acid content of vegetable fiber. Analytical Biochemistry, 96(2), 282-292.

Shahidi, F., Arachchi, J. K. V., \& Jeon, Y.-J. (1999). Food applications of chitin and chitosans. Trends in Food Science E' Technology, 10(2), 37-51.

Shu, S., Sun, C., Zhang, X., Wu, Z., Wang, Z., \& Li, C. (2010). Hollow and degradable polyelectrolyte nanocapsules for protein drug delivery. Acta Biomaterialia, 6(1) 210-217.

Sun, B., Mutch, S. A., Lorenz, R. M., \& Chiu, D. T. (2005). Layered polyelectrolyte-silica coating for nanocapsules. Langmuir, 21(23), 10763-10769.

Szabo, M., Idițoiu, C., Chambre, D., \& Lupea, A. (2007). Improved DPPH determination for antioxidant activity spectrophotometric assay. Chemical Papers, 61(3) 214-216.

Szczepanowicz, K., Hoel, H. J., Szyk-Warszynska, L., Bielaníska, E., Bouzga, A. M. Gaudernack, G., Simon, C., \& Warszynski, P. (2010). Formation of biocompatible nanocapsules with emulsion core and pegylated shell by polyelectrolyte multilayer adsorption. Langmuir, 26(15), 12592-12597.

Wang, J., Zhang, Q., Zhang, Z., Song, H., \& Li, P. (2010). Potential antioxidant and anticoagulant capacity of low molecular weight fucoidan fractions extracted from Laminaria japonica. International Journal of Biological Macromolecules, 46(1), 6-12.

Yamanaka, K. \& Hamano, Y. (2010). Biotechnological production of poly-epsilonL-lysine for food and medical applications. In Y. Hamano (Ed.), Amino-acid homopolymers occurring in nature (Vol. 15) (pp. 61-75). Berlin/Heidelberg: Springer.

Ye, S, Wang C , Liu, X., \& Tong, Z (2005). Multilayer nanocapsules of polysaccharide chitosan and alginate through layer-by-layer assembly directly on PS nanoparticles for release. Journal of Biomaterials Science, Polymer Edition, 16 909-923.

Yoshida, T., \& Nagasawa, T. (2003). e-Poly-L-lysine: Microbial production, biodegradation and application potential. Applied Microbiology and Biotechnology, 62(1), 21-26. 